\title{
Second primary oral squamous cell carcinoma after radiotherapy: a retrospective cohort study
}

\author{
Hao Song ${ }^{1,2 \#}$, Ranran Yang ${ }^{3,4 \#}$, Kailiu Wu ${ }^{1,2}$, Chao Lou ${ }^{1,2}$, Meng Xiao ${ }^{1,2}$, Wei Guo ${ }^{1,2}$, Guoxin Ren ${ }^{1,2}$ \\ ${ }^{1}$ Department of Oral Maxillofacial-Head and Neck Oncology, Shanghai Ninth People's Hospital, Shanghai Jiao Tong University School of Medicine, \\ Shanghai, China; ${ }^{2}$ Shanghai Key Laboratory of Stomatology \& Shanghai Research Institute of Stomatology, National Clinical Research Center of \\ Stomatology, Shanghai, China; ${ }^{3}$ Department of stomatology, Shanghai Ninth People's Hospital, Shanghai Jiao Tong University School of Medicine, \\ Shanghai, China; ${ }^{4}$ Weifang Medical University, Weifang, China \\ Contributions: (I) Conception and design: H Song, G Ren; (II) Administrative support: W Guo; (III) Provision of study materials or patients: H Song, \\ R Yang; (IV) Collection and assembly of data: R Yang; (V) Data analysis and interpretation: H Song, K Wu, C Lou, M Xiao; (VI) Manuscript writing: \\ All authors; (VII) Final approval of manuscript: All authors. \\ \#These authors contributed equally to this work. \\ Correspondence to: Wei Guo; Guoxin Ren. 639 Zhi Zao Ju Road, Shanghai 200011, China. Email: guoweicnsh@163.com; renguoxincnsh@163.com.
}

Background: The purpose of the present study was to investigate the clinicopathological characteristics and prognostic factors of second primary oral squamous cell carcinoma after radiotherapy for head and neck cancer.

Methods: The clinicopathological characteristics of 48 second primary oral squamous cell carcinoma patients with a history of radiotherapy for head and neck cancer were retrospectively analyzed by KaplanMeier survival analysis and Cox proportional hazards model, including gender, age, alcohol consumption, smoking, clinical stage, margin status, regional lymph node status, tumor differentiation and treatment mode.

Results: The second primary oral squamous cell carcinoma mostly occurred on the tongue [18/48], buccal [12/48] and gingiva [10/48], and the 3- and 5-year overall survival (OS) was $60.3 \%$ and 39.4\%, respectively. Margin status and extranodal extension were significantly associated with OS, while only margin status was found to be an independent prognostic factor of OS in the Cox proportional hazards model $(\mathrm{P}=0.003, \mathrm{HR}$ $=3.976,95 \%$ CI: $1.596-9.904)$.

Conclusions: Oral squamous cell carcinoma patients underwent radiotherapy for head and neck cancer show poor survival outcomes. Margin status is an independent prognostic factor of second primary oral squamous cell carcinoma.

Keywords: Second primary; malignancy; squamous cell carcinoma; oral cavity; radiation

Submitted Feb 14, 2021. Accepted for publication May 06, 2021.

doi: $10.21037 /$ tcr-21-283

View this article at: https://dx.doi.org/10.21037/tcr-21-283

\section{Introduction}

Radiotherapy is an essential treatment modality for various malignant head and neck tumors such as nasopharyngeal carcinoma (NPC) and oral squamous cell carcinoma (OSCC) (1-4). However, some patients underwent radiotherapy may develop second malignant tumors in the oral and maxillofacial region, whose clinicopathological characteristics are quite different from that of sporadic oral cancer (5-8). Although many organs are exposed to highor moderate-dose radiation in the radiotherapy for head and neck cancer, the incidence of second malignant tumors varies substantially among different sites. For example, few second tumors are derived from thyroid and salivary gland (1), and the most common site is the oral cavity, in which squamous cell carcinoma was the most common 
histologic type $(1,9)$. A recent study has revealed that NPC survivors with second OSCC had worse prognosis than those with sporadic lesions (5). The nasopharynx, oral cavity and oropharynx are particularly susceptible to radiation-induced tumorigenesis. However, there are few studies on the second primary OSCC in patients underwent radiotherapy for head and neck tumor, and its clinicopathological characteristics remains unclear.

The present study aims to analyze the clinicopathological characteristics and prognostic factors of second primary OSCC after radiotherapy for head and neck cancer. We present the following article in accordance with the STROBE reporting checklist (available at https://dx.doi. org/10.21037/tcr-21-283).

\section{Methods}

\section{Patients and clinical data}

The present study was a retrospective study on second primary OSCC patients with a history of radiotherapy involving oral cavity. The clinical, pathological and therapeutic data and of all OSCC patients with a history of radiotherapy for head and neck tumor in Shanghai Ninth People's Hospital from Dec 2012 to Jan 2015 were retrospectively reviewed. Patients were included if they met the following criteria described by Cahan et al. and Fu et al. $(6,10)$. In brief: (I) they previously underwent radiotherapy for head and neck cancer; (II) the sites of secondary tumor were not adjacent to the first primary OSCC; and (III) there was a period of at least 6 months from the surgery to the diagnosis of second primary tumor. All tumors were confirmed as squamous cell carcinoma in pathology. The study was conducted in accordance with the Declaration of Helsinki (as revised in 2013). The retrospective study was approved by Institutional Research Ethics Committee of Shanghai Ninth People's Hospital (SH9H-2020-TK471-1) and the informed consent was waived.

The clinicopathological characteristics of patients, including gender, age, alcohol consumption, smoking, clinical stage, margin status, regional lymph node status, tumor differentiation and treatment mode, were analyzed by Kaplan-Meier survival analysis [overall survival (OS) and disease-free survival (DFS)], and significant prognostic factors of OS were determined by the Cox proportional hazards model.

Cancer stage was determined according to the 8th criteria of the American Joint Committee on Cancer
(AJCC) (11). The main therapy for the second primary tumor was surgery alone or comprehensive treatment based on surgery. Alcohol consumption and smoking was reviewed in the history of clinical data. Regional lymph node status and tumor differentiation was assessed by pathological diagnosis if neck dissection was performed. Microscopically clear margins were obtained by surgery and assessed in real time. Positive margins were resected immediately until tumor-free margins were verified histologically. The margin status was recorded as positive if there was an initial cutthrough with an invasive tumor at the surgical margin. Adjuvant therapy was administered to patients with positive margins.

\section{Outcome analysis}

Survival was measured from the date of pathologic diagnosis to the most recent contact or death. Follow-up was carried out every 2-4 months for the first year, every 4-6 months for the second year and then every 6 months. Computed tomography scans of craniomaxillofacial and neck regions and lungs were performed every 6 months. Positron emission computed tomography was performed if there was suspicion of distant metastasis. The patients were followed up until April 2020.

\section{Statistical analysis}

Statistical analyses were performed by PASW Statistics 18.0.0 (IBM SPSS Statistics, RRID: SCR 019096). The OS was measured from the date of pathologic diagnosis of the second primary tumor to the most recent follow-up or death. Patients still alive until April 2020 or lost in follow up were censored. The clinical and pathological predictors of OS, including age, gender, alcohol consumption, smoking, clinical stage, margin status, lymph node metastasis, extranodal extension (ENE), and treatment mode, were evaluated by Kaplan-Meier survival analysis and log-rank test, and multivariate analysis controlling for other factors was performed by the Cox proportional hazards model.

\section{Results}

A total of 48 patients (32 males and 16 females) with complete clinical data were enrolled in the study. Their ages ranged from 32 to 81 years with a median of 61 years at diagnosis, and OSCC mostly occurred on the tongue 

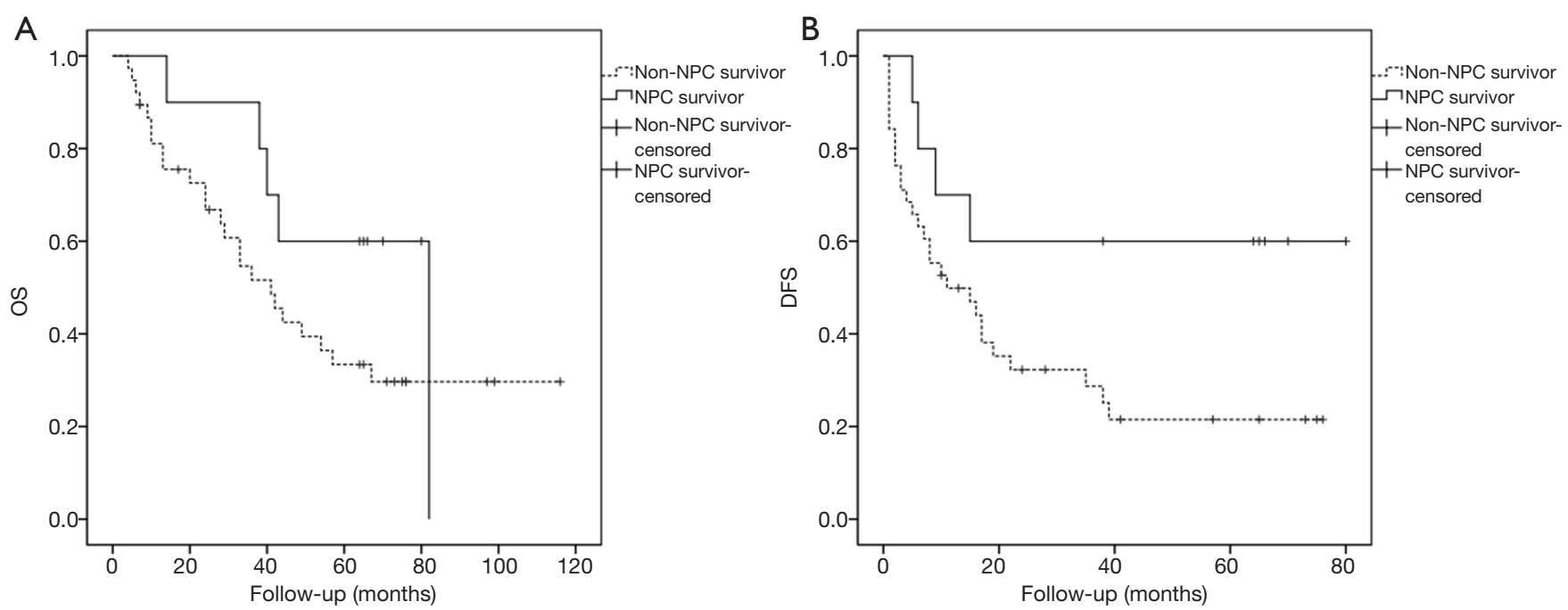

Figure 1 OS and DFS of NPC survivors and non-NPC survivors. (A) OS of NPC survivors and non-NPC survivors showed no significant difference. (B) NPC survivors showed a trend of better DFS than non-NPC survivors, but the difference showed no significance ( $\mathrm{P}=0.055)$. OS, overall survival; NPC, nasopharyngeal carcinoma; DFS, disease free survival.

[18/48], buccal [12/48] and gingiva [10/48]. Of these 48 patients, 10 were NPC survivors and 38 were non-NPC survivors, and the primary tumor was located in the head and neck region and treated with radiotherapy. NPC survivors had higher, albeit not statistically significant, DFS ( $\mathrm{P}=0.069, \mathrm{HR}=0.377,95 \%$ CI: 0.132-1.080) and OS $(\mathrm{P}=0.216, \mathrm{HR}=0.543,95 \% \mathrm{CI}: 0.207-1.428)$ than nonNPC survivors (Figure 1). The demographic and clinical characteristics of the 48 patients were shown in Table 1. The follow-up ranged from 4 to 116 months with a median of 40.5 months, and two patients were lost in follow-up. Twenty-nine patients were dead during the follow-up. The most frequent sites of the first primary tumor were the oral cavity $(\mathrm{n}=27)$, nasopharynx $(\mathrm{n}=10)$ and oropharynx $(\mathrm{n}=3)$.

\section{Treatment}

The second primary OSCC was treated primarily by surgery. Complete resection was performed in all patients with initial cut-through with an invasive tumor at the surgical margin $(n=9)$. Of these 48 patients, 37 received surgery alone and 11 received comprehensive treatment based on surgery, in which 10 received surgery combined with chemotherapy and 1 received surgery combined with chemoradiotherapy. There was no significant difference in $\mathrm{OS}(\mathrm{P}=0.908, \mathrm{HR}=0.948,95 \%$ CI: $0.383-2.345)$ and
DFS $(\mathrm{P}=0.880, \mathrm{HR}=0.937,95 \%$ CI: $0.405-2.170)$ between patients received surgery only and those patients received comprehensive treatment.

\section{Prognostic factors}

The 3 - and 5 -year OS was $60.3 \%$ and $39.4 \%$, and the 1-, 2-, 3 - and 5 -year DFS was $75.0 \%, 38.3 \%, 35.8 \%$ and $30.4 \%$, respectively. The prognostic factors of OS and DFS in patients with second OSCC were shown in Table 2. Margin status $(\mathrm{P}=0.002, \mathrm{HR}=4.033,95 \% \mathrm{CI}: 1.643-9.899)$ and ENE $(\mathrm{P}=0.050, \mathrm{HR}=2.765,95 \% \mathrm{CI}: 0.954-8.012)$ were significantly associated with $\mathrm{OS}$; and margin status was also significantly associated with DFS $(\mathrm{P}=0.002, \mathrm{HR}=3.538$, 95\% CI: $1.608-7.782)$. However, age $(\mathrm{P}=0.151, \mathrm{HR}=0.582$, 95\% CI: 0.274-1.234), gender $(\mathrm{P}=0.519, \mathrm{HR}=0.783,95 \%$ CI: 0.371-1.652), alcohol consumption $(\mathrm{P}=0.388$, HR $=1.886$, 95\% CI: 0.447-7.962), smoking $(\mathrm{P}=0.161$, HR $=2.138,95 \%$ CI: $0.740-6.183)$, clinical stage $(\mathrm{P}=0.497$, $\mathrm{HR}=1.345,95 \% \mathrm{CI}: 0.571-3.167)$, lymph node metastasis $(\mathrm{P}=0.866, \mathrm{HR}=1.071,95 \% \mathrm{CI}: 0.483-2.377)$ and tumor differentiation ( $\mathrm{P}=0.505, \mathrm{HR}=1.434,95 \%$ CI: 0.497-4.141) were not significantly associated with OS. The multivariate analysis showed that only margin status was a significant independent prognostic factor of OS $(\mathrm{P}=0.003, \mathrm{HR}=3.976$, 95\% CI: 1.596-9.904) (Figure 2). 
Table 1 Demographic and clinicopathological data of 48 patients with second primary OSCC

\begin{tabular}{|c|c|}
\hline Characteristic & No. of patients \\
\hline \multicolumn{2}{|l|}{ Age } \\
\hline$\geq 60$ years & 25 \\
\hline$<60$ years & 23 \\
\hline \multicolumn{2}{|l|}{ Gender } \\
\hline Male & 32 \\
\hline Female & 16 \\
\hline \multicolumn{2}{|l|}{ Drinker } \\
\hline Yes & 7 \\
\hline No & 41 \\
\hline \multicolumn{2}{|l|}{ Smoker } \\
\hline Yes & 12 \\
\hline No & 36 \\
\hline \multicolumn{2}{|l|}{ Tumor site } \\
\hline Tongue & 18 \\
\hline Gingiva & 10 \\
\hline Buccal & 12 \\
\hline Floor of mouth & 4 \\
\hline Palate & 3 \\
\hline Mandibular bone & 1 \\
\hline \multicolumn{2}{|l|}{ Clinical stage } \\
\hline I & 5 \\
\hline II & 8 \\
\hline III & 8 \\
\hline IV & 27 \\
\hline \multicolumn{2}{|l|}{ Tumor stage } \\
\hline $\mathrm{T} 1$ & 6 \\
\hline $\mathrm{T} 2$ & 10 \\
\hline T3 & 6 \\
\hline $\mathrm{T} 4$ & 26 \\
\hline \multicolumn{2}{|l|}{ Status of lymph node } \\
\hline NO & 36 \\
\hline $\mathrm{N}+$ & 12 \\
\hline \multicolumn{2}{|l|}{ Differentiation } \\
\hline Well or moderate & 43 \\
\hline Poor & 5 \\
\hline \multicolumn{2}{|l|}{ Margin status } \\
\hline Positive & 9 \\
\hline Negative & 39 \\
\hline \multicolumn{2}{|l|}{ Treatment mode } \\
\hline Surgery only & 37 \\
\hline Surgery + adjuvant therapy & 11 \\
\hline
\end{tabular}

OSCC, oral squamous cell carcinoma.

\section{Discussion}

The present study has revealed that secondary OSCC patients underwent radiotherapy for head and neck cancer show poor survival outcomes, and the prognostic of squamous cell carcinoma in oral cavity with a history of radiotherapy was not in accordance with its sporadic counterpart. Margin status is an independent prognostic factor of second primary OSCC.

Complete resection with tumor-free margins is a key goal of oncologic surgery. However, our clinical experience has suggested that it is difficult to identify tumor border in OSCC patients with a history of radiotherapy due to changes in the soft tissue and chronic oral mucosal inflammation after radiotherapy. Occasionally, there is an initial cut-through of the invasive tumor at the surgical margin. Although additional adjacent tumor-free margins are achieved, the risk of local relapse is still high (12). In the present study, additional adjacent tumor-free margins are achieved in all cases, and margin status is found to be an independent prognostic factor of OS in patients developed second primary OSCC and it is also significantly associated with DFS. Thus, it is important to determine the border of the secondary tumor and achieve a clear margin in surgery in these patients.

The presence or absence of ENE is used to assign $\mathrm{N}$ category in AJCC 8th edition in head and neck SCC. ENE is defined as tumor extension through the lymph node capsule into surrounding connective tissues (13). In our study, ENE is found to be significantly associated with OS.

Lifestyle factors such as tobacco and alcohol abuse may have a negative impact on OS $(14,15)$. In this study, there is no significant association between lifestyle factors (alcohol consumption and smoking) and the survival of patients. However, it is important to note that we could not obtain an accurate record of smoking in pack-years and the number of alcohol consumption days per week or the amount of drinking per day.

Ionizing radiation is a well-known risk factor of malignant tumors. Previous studies on second primary tumors in the head and neck region induced by radiotherapy have mainly focused on NPC survivors. However, we have also found in clinical practice that patients underwent radiotherapy for head and neck cancer could have tumors in the oral cavity, major salivary gland, and nasal cavity. Thus, non-NPC survivors should also be considered in investigating the outcomes and clinicopathological characteristics of second primary OSCC. A recent study 
Table 2 Prognostic factors for the OS and DFS in patients with secondary OSCC

\begin{tabular}{|c|c|c|c|c|c|c|c|c|c|c|c|}
\hline \multirow{3}{*}{ Parameters } & \multirow{3}{*}{$\begin{array}{l}\text { No. of } \\
\text { case }\end{array}$} & \multirow{3}{*}{$\begin{array}{l}\text { 5-year } \\
\text { OS (\%) }\end{array}$} & \multicolumn{5}{|c|}{ Cox regression } & \multirow{3}{*}{$\begin{array}{c}\text { 3-year } \\
\text { DFS (\%) }\end{array}$} & \multirow{3}{*}{ Log-rank- } & \multirow{2}{*}{\multicolumn{2}{|c|}{$\begin{array}{c}\text { Cox regression } \\
\text { Univariate }\end{array}$}} \\
\hline & & & \multirow[b]{2}{*}{$P$} & \multicolumn{2}{|c|}{ Univariate } & \multicolumn{2}{|c|}{ Multivariate } & & & & \\
\hline & & & & $\begin{array}{c}\text { HR } \\
(95 \% \mathrm{Cl})\end{array}$ & $P$ & $\begin{array}{c}\text { HR } \\
(95 \% \mathrm{Cl})\end{array}$ & $\mathrm{P}$ & & & $\begin{array}{c}\text { HR } \\
(95 \% \mathrm{Cl})\end{array}$ & $\mathrm{P}$ \\
\hline Age & & & 0.151 & 0.582 & 0.151 & - & - & & 0.934 & 0.972 & 0.936 \\
\hline$\geq 60$ years & 25 & 49.0 & & $(0.274-1.234)$ & & & & 35.6 & & $(0.485-1.947)$ & \\
\hline Gender & & & 0.519 & 0.783 & 0.519 & - & - & & 0.903 & 0.957 & 0.905 \\
\hline Male & 32 & 45.8 & & $(0.371-1.652)$ & & & & 39.3 & & $(0.467-1.961)$ & \\
\hline Female & 16 & 27.3 & & & & & & 30.0 & & & \\
\hline Smoker & & & 0.149 & 2.138 & 0.161 & - & - & & 0.650 & 1.209 & 0.657 \\
\hline Yes & 12 & 62.9 & & $(0.740-6.183)$ & & & & 38.9 & & $(0.523-2.798)$ & \\
\hline No & 36 & 32.9 & & & & & & 34.6 & & & \\
\hline Clinical stage & & & 0.494 & 1.345 & 0.497 & - & - & & 0.929 & 1.035 & 0.931 \\
\hline$I+I I$ & 13 & 50.8 & & $(0.571-3.167)$ & & & & 38.5 & & $(0.478-2.238)$ & \\
\hline III + IV & 35 & 35.0 & & & & & & 34.8 & & & \\
\hline Margin status & & & 0.001 & 4.033 & 0.002 & 3.976 & 0.003 & & 0.001 & 3.538 & 0.002 \\
\hline ENE & & & 0.050 & 2.765 & 0.061 & 2.622 & 0.080 & & 0.410 & 1.626 & 0.426 \\
\hline+ & 4 & 40.8 & & $(0.954-8.012)$ & & $(0.891-7.721)$ & & 37.0 & & $(0.491-5.387)$ & \\
\hline- & 44 & 25.0 & & & & & & 25.0 & & & \\
\hline Differentiation & & & 0.501 & 1.434 & 0.505 & - & - & & 0.814 & 0.869 & 0.818 \\
\hline Poor & 5 & 40.0 & & $(0.497-4.141)$ & & & & 40.0 & & $(0.265-2.857)$ & \\
\hline Well \& moderate & 43 & 39.4 & & & & & & 35.2 & & & \\
\hline Treatment mode & & & 0.907 & 0.948 & 0.908 & - & - & & 0.878 & 0.937 & 0.880 \\
\hline Surgical only & 37 & 39.3 & & (0.383-2.345) & & & & 39.3 & & $(0.405-2.170)$ & \\
\hline Comprehensive & 11 & 40.0 & & & & & & 40.0 & & & \\
\hline
\end{tabular}

OS, overall survival; DFS, disease-free survival; HR, hazard ratio; ENE, extra-nodal extension. 

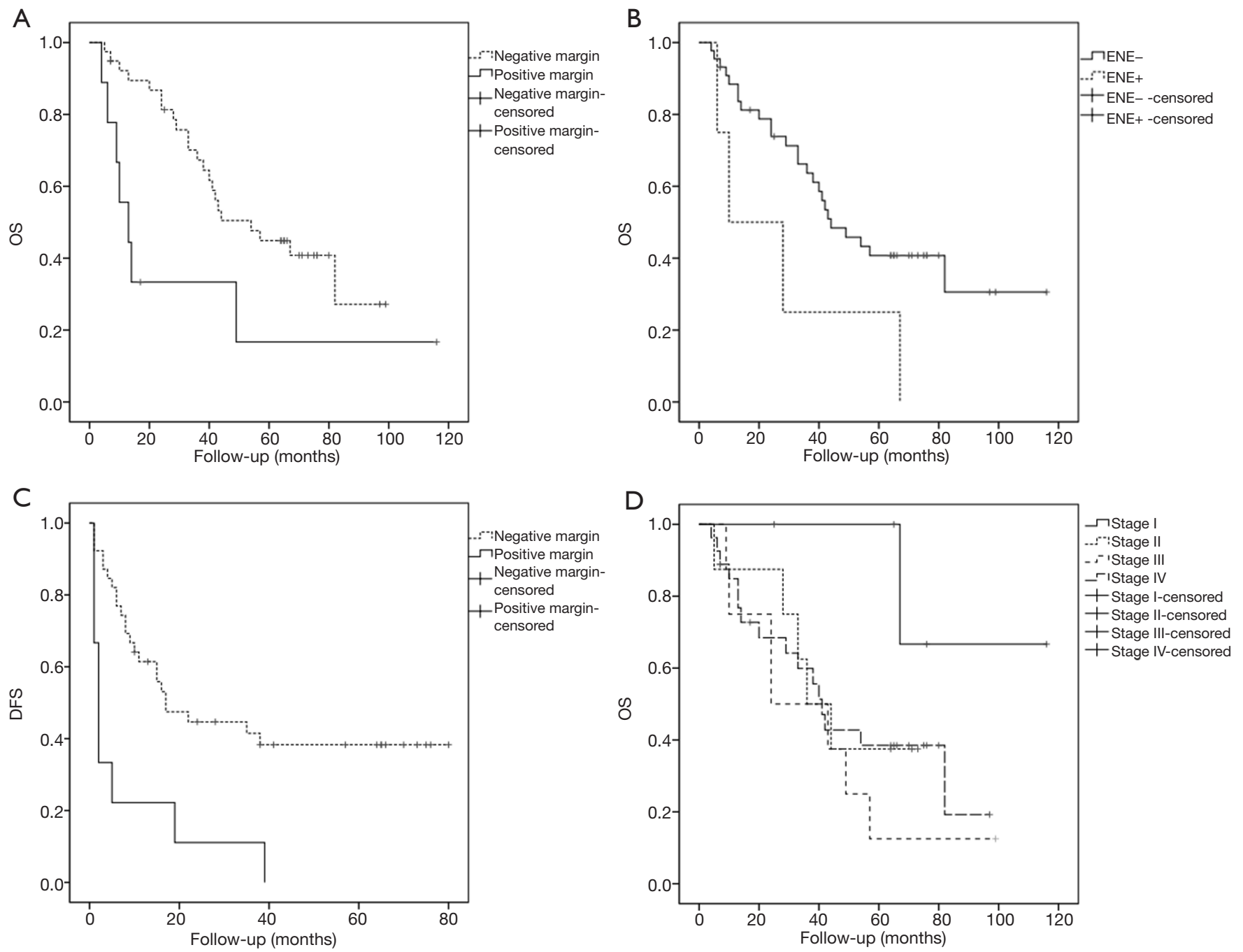

Figure 2 Prognostic factors of second OSCC. Kaplan-Meier analysis of 48 patients with second OSCC by margin status (A,C), ENE (B) and clinical stage (D). The log-rank test showed that patients with positive margin had a worse OS (A) and DFS (C), and patients with ENE+ had a worse OS (B). While clinical stage was not significantly associated with OS (D). OSCC, oral squamous cell carcinoma; ENE, extranodal extension; OS, overall survival; DFS, disease free survival.

including some non-NPC survivors in second primary tumor patients has focused only on specific gingival squamous cell carcinoma (6). To the best of our knowledge, this is the first study on the prognosis of second primary OSCC in both NPC and non-NPC survivors with a history of radiotherapy for head and neck cancer.

It has been reported that the survival outcome is better in patients with sporadic oral cancer than in patients with a history of radiotherapy for head and neck cancer. Zhang et al. (7) and Sun et al. (16) investigated the prognosis of tongue squamous cell carcinoma with a history of radiotherapy for NPC. Dai et al. (5) showed that second primary OSCC had a worse outcome than sporadic OSCC in NPC survivors. In the present study, the 5-year OS of patients with secondary OSCC is $39.4 \%$, which is much worse than those with sporadic lesions reported in literature.

It is also noted that the sites differ between sporadic oral cancer and second primary tumor in patients with a history of radiotherapy. The most frequent sites of second primary tumor are the tongue, gingiva and buccal, while those of sporadic lesions are the tongue, gingiva and hard plate (6). 
We have found that the prognosis of second primary oral cancer is not in accordance with the clinical stage of AJCC staging system on OSCC. Patients with stage II, III and IV lesions show similar OS. The patients with clinical stage I had a trend of better prognosis than other stages (significance was reached comparing stage I and III, $\mathrm{P}=0.018$ ). Fu et al. (6) found that some clinicopathologic characteristics, such as prominent sites and TNM stage, were significant prognostic factors of second primary gingival squamous cell carcinoma after radiotherapy. Thus, the prognostic factor of secondary OSCC may be different from that of sporadic OSCC.

It is difficult to select the cases with secondary tumors caused by radiotherapy and make critical distinction between tumors caused by radiotherapy and sporadic tumors arising after radiotherapy. In all of the previous investigations on secondary malignant tumors, there was no exact method to distinguish secondary tumors from sporadic except by criteria based on reviewing the history of radiation. Although it is difficult to make critical distinction of tumor caused by radiotherapy and tumor can occur without radiotherapy involved, it is important to investigate the difference between malignancy with and without a history of radiotherapy in oral and maxillofacial region. In the present study, we analyzed the patients with a history of radiotherapy involving the region of secondary tumors, following the criteria established by previous investigators of selecting the secondary tumors with the greatest extent possible.

Previous studies have shown no significant difference in OS between patients received surgery and those received comprehensive treatment based on surgery $(5,16)$. There might be a bias, for comprehensive treatment was much more probable considered when the tumor was in more advanced stage. In this study, no significant improvement is achieved in patients received comprehensive treatment based on surgery. It has been reported that surgery was superior to non-surgical treatment mode in second primary SCC after radiation in head and neck region. Systematic treatment in this study is the platinum-based chemotherapy. Given the poor prognosis of second primary OSCC, there is a need to find new treatments to improve the survival.

The use of intensity-modulated radiation therapy (IMRT) for head and neck tumor has been increasing in recent years, as it has the potential to deliver complex dose distributions to avoid critical structures close to the target. However, concerns exist about whether the risk of second cancer after radiotherapy could be reduced. However, Ardenfors et al. (17) investigated whether IMRT and 3-dimensional conformal radiotherapy (3D-CRT) could reduce the risk of second tumors induced by radiotherapy for head and neck tumor. The results showed that the redistribution of the dose characteristic to IMRT only leads to a redistribution of risks in individual tissue, but no difference in total levels of risk was found between the irradiation techniques considered.

The mechanism of the difference between secondary OSCC and sporadic SCC remains unknown. The poor outcome may be attributed to Bmil upregulation in the secondary lesions (18). Patients often present with chronic oral mucosal inflammation after radiotherapy, but it remains unknown whether such inflammation could develop to carcinoma. The mechanism of second primary OSCC requires further investigation.

\section{Conclusions}

Secondary OSCC patients underwent radiotherapy for head and neck cancer show poor survival outcomes, and margin status is an independent prognostic factor of second primary OSCC.

\section{Acknowledgments}

Funding: This work was supported by the Interdisciplinary Program of Shanghai Jiao Tong University (YG2021QN59) and Fundamental research program funding of Ninth People's Hospital affiliated to Shanghai Jiao Tong University School of Medicine (JYZZ04).

\section{Footnote}

Reporting Checklist: The authors have completed the STROBE reporting checklist. Available at https://dx.doi. org/10.21037/tcr-21-283

Data Sharing Statement: Available at https://dx.doi. org/10.21037/tcr-21-283

Conflicts of Interest: All authors have completed the ICMJE uniform disclosure form (available at https://dx.doi. org/10.21037/tcr-21-283). The authors have no conflicts of interest to declare.

Ethical Statement: The authors are accountable for all aspects of the work in ensuring that questions related 
to the accuracy or integrity of any part of the work are appropriately investigated and resolved. The study was conducted in accordance with the Declaration of Helsinki (as revised in 2013). The retrospective study was approved by Institutional Research Ethics Committee of Shanghai Ninth People's Hospital (SH9H-2020-TK471-1) and the informed consent was waived.

Open Access Statement: This is an Open Access article distributed in accordance with the Creative Commons Attribution-NonCommercial-NoDerivs 4.0 International License (CC BY-NC-ND 4.0), which permits the noncommercial replication and distribution of the article with the strict proviso that no changes or edits are made and the original work is properly cited (including links to both the formal publication through the relevant DOI and the license). See: https://creativecommons.org/licenses/by-nc-nd/4.0/.

\section{References}

1. Xi M, Liu SL, Zhao L, et al. Prognostic factors and survival in patients with radiation-related second malignant neoplasms following radiotherapy for nasopharyngeal carcinoma. PLoS One 2013;8:e84586.

2. Liao LJ, Chou HW, Wang CT, et al. The impact of second primary malignancies on head and neck cancer survivors: a nationwide cohort study. PLoS One 2013;8:e62116.

3. Lin C, Lin SW, Weng SF, et al. Risk of second primary malignancies after nasopharyngeal carcinoma: a population-based cohort study in Taiwan. Head Neck 2014;36:209-14.

4. Chan JY, Gooi Z, Mydlarz WK, et al. Risk of second primary malignancy after nasopharyngeal carcinoma in the United States: A population-based study. Head Neck 2016;38 Suppl 1:E1130-6.

5. Dai L, Fang Q, Li P, et al. Secondary Squamous Cell Carcinoma of the Oral Cavity after Nasopharyngeal Carcinoma. Cancer Res Treat 2020;52:109-16.

6. Fu X, Chen S, Chen W, et al. Clinical analysis of second primary gingival squamous cell carcinoma after radiotherapy. Oral Oncol 2018;84:20-4.

7. Zhang P, Zhang L, Liu H, et al. Clinicopathologic Characteristics and Prognosis of Tongue Squamous Cell Carcinoma in Patients with and without a History of Radiation for Nasopharyngeal Carcinoma: A Matched Case-Control Study. Cancer Res Treat 2017;49:695-705.

8. Song M, Zhuang SM, Chen SW, et al. Survival study and treatment strategy for second primary tumors in the oral cavity in patients with nasopharyngeal carcinoma after definitive radiation. Head Neck 2012;34:1551-5.

9. Teshima T, Inoue T, Chatani M, et al. Incidence of other primary cancers in 1,569 patients with pharyngolaryngeal cancer and treated with radiation therapy. Strahlenther Onkol 1992;168:213-8.

10. Cahan WG, Woodard HQ, Higinbotham NL, et al. Sarcoma arising in irradiated bone: report of eleven cases. 1948. Cancer 1998;82:8-34.

11. Amin MB, Edge S, Greene F, et al. editors. AJCC Cancer Staging Manual. 8th edition. Chicago, IL: Springer, 2017.

12. Scholl P, Byers RM, Batsakis JG, et al. Microscopic cutthrough of cancer in the surgical treatment of squamous carcinoma of the tongue. Prognostic and therapeutic implications. Am J Surg 1986;152:354-60.

13. Wreesmann VB, Katabi N, Palmer FL, et al. Influence of extracapsular nodal spread extent on prognosis of oral squamous cell carcinoma. Head Neck 2016;38 Suppl 1:E1192-9.

14. Liu B, Shen M, Xiong J, et al. Synergistic effects of betel quid chewing, tobacco use (in the form of cigarette smoking), and alcohol consumption on the risk of malignant transformation of oral submucous fibrosis (OSF): a case-control study in Hunan Province, China. Oral Surg Oral Med Oral Pathol Oral Radiol 2015;120:337-45.

15. Lee CH, Ko YC, Huang HL, et al. The precancer risk of betel quid chewing, tobacco use and alcohol consumption in oral leukoplakia and oral submucous fibrosis in southern Taiwan. Br J Cancer 2003;88:366-72.

16. Sun $\mathrm{C}, \mathrm{Hu} \mathrm{Z}$, Zhong Z, et al. Clinical and prognostic analysis of second primary squamous cell carcinoma of the tongue after radiotherapy for nasopharyngeal carcinoma. Br J Oral Maxillofac Surg 2014;52:715-20.

17. Ardenfors O, Josefsson D, Dasu A. Are IMRT treatments in the head and neck region increasing the risk of secondary cancers? Acta Oncol 2014;53:1041-7.

18. Hu Q, Wu T, Chen X, et al. The poor outcome of second primary oral squamous cell carcinoma is attributed to Bmi1 upregulation. Cancer Med 2018;7:1056-69.

Cite this article as: Song $\mathrm{H}$, Yang $\mathrm{R}, \mathrm{Wu} \mathrm{K}$, Lou C, Xiao M, Guo W, Ren G. Second primary oral squamous cell carcinoma after radiotherapy: a retrospective cohort study. Transl Cancer Res 2021;10(6):2747-2754. doi: 10.21037/tcr-21-283 